\title{
Waste Pharmaceutical Blister Packages as a Source of Secondary Aluminum
}

\author{
SUGAM SHUKLA, ${ }^{1}$ PETTERI HALLI, ${ }^{1}$ MUHAMMAD KAMRAN KHALID, ${ }^{1}$ \\ and MARI LUNDSTRÖM ${ }^{1,2}$ \\ 1.-Department of Chemical and Metallurgical Engineering (CMET), School of Chemical \\ Engineering, Aalto University, Vuorimiehentie $2 \mathrm{~K}, 02150$ Espoo, Finland. \\ 2.—e-mail: mari.lundstrom@aalto.fi
}

\begin{abstract}
Waste pharmaceutical blister packages (WPBs) are a source of solid waste, which are composed of plastics and aluminum, therefore acting as a potential source for secondary aluminum. The structure of WPBs makes the recycling of aluminum notably more complex than typical aluminum recycling. Currently, WBPs are disposed of as municipal solid waste; thus, aluminum is lost from the circulation during incineration. In this work, three types of WPBs were studied, each with two plastic layers and a metallic layer. Delamination of WPBs to separate aluminum and plastic(s) was investigated by using a solution of organic solvents. The effects of temperature $\left(30-50^{\circ} \mathrm{C}\right)$, acetone to isopropanol ratio (0-100 vol.\%) and different types of WPBs on delamination behavior were investigated. The results suggest that aluminum separation and recovery from WPBs is $100 \%$ at optimum conditions. Moreover, an overall indicative flowsheet for recycling and post-processing of segregated aluminum from the plastic is also suggested.
\end{abstract}

\section{INTRODUCTION}

The packaging industry is one of the largest industrial verticals in the world, with an overall value of $€ 250$ billion. ${ }^{1}$ In the 1960 s, packaging of solid pharmaceuticals such as tablets and capsules in blister packaging became popular. Compared with other alternatives such as plastic and glass bottles, blister packaging exhibits better sealing properties. ${ }^{2}$ Other benefits of blister packages have been suggested, such as product integrity, product protection, being tamper proof, reduced possibility of accidental misuse and patient compliance. ${ }^{1}$ However, the increase in environmental concern, not only about the generation of medical and pharmaceutical waste, but also the waste generated from packaging material and its handling, has resulted in strict EU rules and regulations. ${ }^{3}$ Globally, the packaging of pharmaceuticals and drugs accounts for approximately $4 \%$ of the whole packaging industry. In Europe, nearly 85\% of drugs in the form of

(Received July 29, 2021; accepted November 9, 2021; published online January 12, 2022) tablets are packed in blister packaging. ${ }^{1}$ These blisters not only provide an efficient physical protection for the drugs but also a barrier in terms of moisture, light and/or oxygen. Currently, the handling of waste pharmaceutical blisters (WBPs) is done by either using landfill or treating them in incinerators. ${ }^{4}$ The main drawback of the incineration process is the loss of aluminum from circulation along with its oxidation. ${ }^{5}$ In addition, the generation of toxic gases can occur.

Generally, pharmaceutical blister packages are composed of various plastics and metallic foil, as shown in Fig. 1. This type of structure, consisting of aluminum (lidding material) and plastics (forming film), such as polyvinyl chloride PVC, polypropylene $(\mathrm{PP})$, polystyrene (PS) and/or polyethylene (PE), makes the recycling of aluminum from WBPs notably more difficult and complex compared to typical aluminum recycling. ${ }^{6,7}$ Two types of WPBs are commonly utilized by the pharmaceutical packaging industry: Type I: aluminum and a single layer of plastic; Type II: aluminum and a multilayer of plastic. 


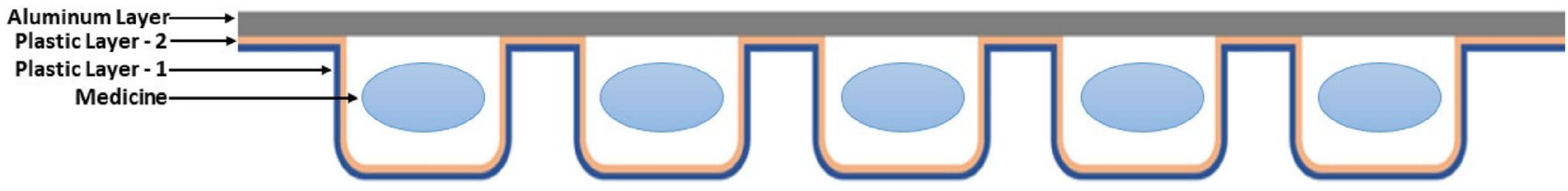

Fig. 1. Schematic of medical blister packages.

The PVC present in WPBs is known to complicate the incineration process. Incineration of PVC with mixed municipal waste may lead to the generation of hazardous pollutants such as nitrous oxide, sulfur oxides, dusts and Cl-containing dioxins. ${ }^{8}$ In addition, the hydrogen chloride gas formed can react with moisture within the incinerator, resulting in the formation of hydrochloric acid. The hydrochloric acid generated during the process can cause damage through corrosion of incinerator refractories, reducing the lifetime of the incinerator. However, when mixed plastic waste including PP, PS, PE along with $\mathrm{PVC}$ is fed into the incinerator, the hydrochloric acid formed during the process reduces the recycling ratio of other plastics because of chemical deterioration. $^{8,9}$

The PVC or chlorine present in waste promotes the cyclization of organics and formation of aromatic compounds during incineration. PVC or chlorine promotes the cyclization of long-chain alkenes through dechlorane reactions and generates aromatic organics, chloro-aromatic hydrocarbons or char. ${ }^{10}$ Under oxygen-deficit conditions, metal chlorides promote the thermal decomposition of PS plastic and subsequently react with benzene, which ultimately favors the formation and growth of chlorinated polycyclic aromatic hydrocarbons (ClPAHs). ${ }^{11}$ These PAH compounds are known to be carcinogenic, mutagenic and teratogenic and may cause cardiovascular disease. ${ }^{12}$

Instead of incineration of plastics such as PP, PS, $\mathrm{PE}$ and PVC, which is already partially restricted in many European countries, mechanical and chemical recycling can provide an advantage due to the generation of few pollutants and wide variety of recycled products. ${ }^{13,14}$ Gente et al. ${ }^{15,16}$ conducted a mechanical separation study by employing cryocomminution on WPBs and further separation of aluminum (conducting fraction) and plastic (nonconducting fraction) through electrostatic separation. The results of their study showed a high aluminum (conductor fraction) recovery rate (approximately $80 \%$ ) and a high plastic (non-conductor fraction) recovery rate (approximately 90\%) in optimal conditions. However, according to the method they suggested in another study, the presence of plastics in the conducting fraction and aluminum in the non-conducting fraction implies that each fraction is still impure and hence requires further treatment. ${ }^{15,16}$ Agarwal et al. ${ }^{5}$ studied the mechanical separation of WPBs into aluminum and plastic fractions by applying electrodynamic fragmentation. The recovery of aluminum was up to $88 \%$ with a purity of approximately $99.4 \% .^{5}$

In contrast to the mechanical route for the separation of the aluminum and plastic fractions, the chemical route has also been studied extensively in recent years. Wang et al. ${ }^{7,17}$ studied the leaching of aluminum from WPBs with two different leaching agents, viz., sodium hydroxide and hydrochloric acid. The results of both the studies, under optimized conditions, showed high recovery rates of aluminum (up to 100\%). However, there are certain drawbacks with the selection of the leaching agents, as both sodium hydroxide and hydrochloric acid are corrosive in nature and can cause harm to the environment. ${ }^{18}$ Moreover, further processing is required to recover aluminum from the pregnant leaching solution, therefore adding further cost to the process. Yousef et al. ${ }^{18}$ investigated the delamination process by using $\mathrm{N}, \mathrm{N}$-dimethyl-cyclohexylamine (DMCHA) as a switchable hydrophilicity solvent along with ultrasonic treatment to enhance the adhesive bonding between the aluminum and plastic in WPBs. The approach suggested by Yousef et al. ${ }^{18}$ consists of several steps, hence complicating the overall process. This leads to an increase in energy consumption and an increase in the cost of the recycling and recovery process of aluminum and plastic. Moreover, the utilization of DMCHA in this approach is not preferred as a sustainable and green process because of its high corrosiveness and toxicity. Therefore, it is considered a safety and environmental hazard. ${ }^{18}$ Finally, Nieminen et al. ${ }^{2}$ presented a study in which they investigated deep eutectic solvents (DES) consisting of lactic acid and choline chloride (molar ratio 1:9) and pure lactic acid for separating aluminum and plastic. However, after pure DES treatment, the recovered aluminum was highly oxidized consisting of 65 wt.\% aluminum and 23 wt.\% oxygen because of the formation of aluminum lactate, whereas after lactic acid treatment, the aluminum foil surface contained $95 \mathrm{wt} . \%$ aluminum. Also, the solvent purification methods and recovery of the dissolved aluminum require further research. ${ }^{2}$

Several organic solvents in various research studies have been investigated for the delamination process and for interaction between the organic solvents and plastics. According to Summers and Rabinovitch, acetone swells the PVC very rapidly but only up to a certain extent and attains an equilibrium after a certain residence time due to the 
penetration of acetone into the amorphous PVC only, without destroying the crystalline PVC, which acts as the crosslink. ${ }^{19}$ Similarly, in another study performed by Papaspyrides and Tingas, ${ }^{20}$ they suggested that isopropanol penetrates PVC with ease and hence has higher rates of sorption than isooctane, therefore facilitating delamination of WPBs. Additionally, the aluminum present in WPBs reacts with neither acetone nor isopropanol under atmospheric conditions. ${ }^{21}$

The purpose of this study is to investigate and develop a hydrometallurgical process to resolve the challenges in recycling of aluminum from waste pharmaceutical blister packs, thereby reducing medical waste. Moreover, the proposed process can be considered environmentally benign as green solvents (acetone and iso-propanol) are used. In addition, the process can provide not only an efficient and sustainable method for the recovery of aluminum but also potentially for plastics from waste without the generation of hazardous pollutants.

\section{EXPERIMENTAL SECTION}

\section{Sample Collection and Types of WPBs}

Samples of different types of WPBs were collected from a machine dose dispenser of Pharmac Finland Oy pharmaceutical company, as shown in Fig. 2. The collected WPB samples were sorted manually into two types based on the color profile, i.e., two samples, A and B: silver-colored WPBs with translucent plastic film (Sample A and) and white-colored WPBs with opaque plastic film (Sample B). Sample $\mathrm{C}$ was used for the verification of parameter optimization experiments. The selection was based on visual appearance; Sample C was a silver-colored WPB. Additionally, Sample C had similar structure, i.e., two plastic layers and one aluminum layer, with poly-acrylic based adhesive.

Sample selection was based on the homogeneity and similarity among all the samples, i.e., Sample
A, B and C. This was ensured by ascertaining that all the Sample As were from the same manufacturer; all the Sample Bs were from the same manufacturer(s) (different from Sample A) and of the same drug. Sample C followed the same pattern.

The differences among the three sample sets, A, B and $\mathrm{C}$, were first that they all were from different manufacturer.s Second, they all were for different drugs and different drug delivery methods, viz., Sample A was in the form of capsules; Sample B and Sample C were tablets. Lastly, the main difference in all three samples was the thickness of the plastic layers and aluminum layer.

\section{Characterization of WPBs}

Fourier transformation infrared spectroscopy (FTIR, Nicolet Magna 380, Smart Orbit, USA) was used to analyze the plastic material present in WPB sample types A and B. A total of 32 scans were completed and integrated for each spectrum at a resolution of $4 \mathrm{~cm}^{-1}$ in the wavenumber range from $400 \mathrm{~cm}^{-1}$ to $4000 \mathrm{~cm}^{-1}$. The cross-section measurement and surface morphology of WPBs were analyzed using scanning electron microscope (SEM, Tescan Mira3 GM, Czech Republic) coupled with energy-dispersive spectroscopy (EDS, UltraDry 50 $\mathrm{mm}^{2}$ EDS detector and NSS software, Thermo Scientific, USA).

The WPB sample preparation for SEM analysis was performed by cutting small pieces of WPB Samples A, B and C using a sharp cutter. These small pieces of WPBs were then cast in epoxy. Once the epoxy containing the samples had hardened, polishing was performed (with $1 \mu \mathrm{m}$ multi-crystal diamonds, Struers, Denmark), and the polished samples together with the epoxy holder were carbon coated (EM SCD050, Leica, Japan) to provide a conductive layer on top of the samples. EDS was also performed on the same epoxy-embedded carbon-coated samples to analyze the metallic and

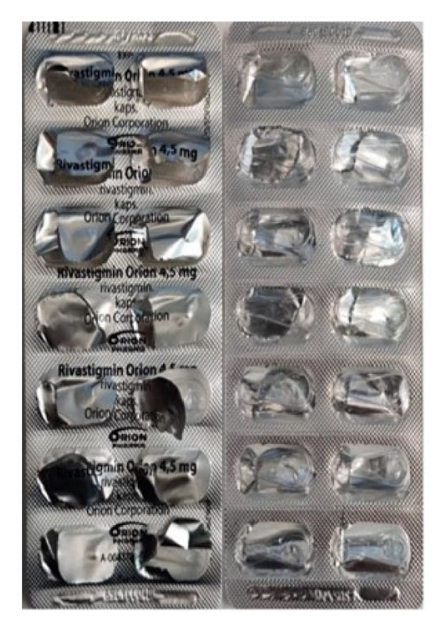

Sample - A

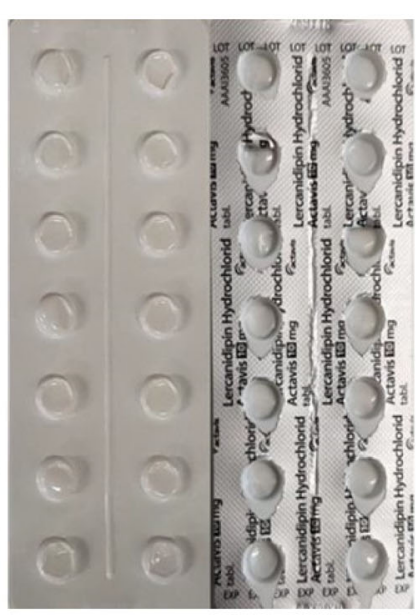

Sample - B

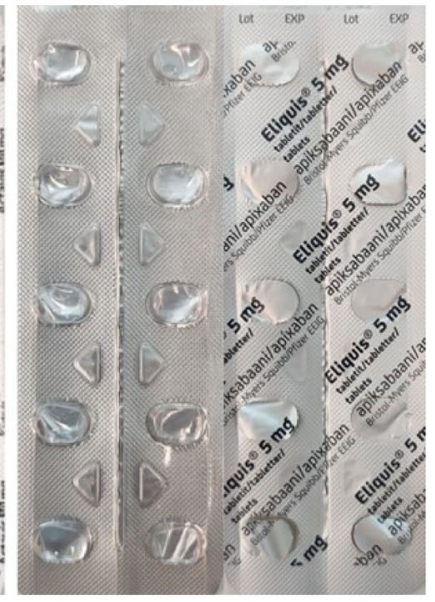

Sample - C

Fig. 2. Types of waste pharmaceutical blisters: Samples A, B and C, used as raw materials in the experiments. 
plastic composition of the lidding material and forming film in WPBs.

\section{Delamination Experiments}

The experiments were performed in batches using $250-\mathrm{ml}$ beakers made of borosilicate glass. Prior to every experiment, all the WPB samples were weighed. The delaminating agents used were acetone $(\mathrm{C} 3 \mathrm{H} 6 \mathrm{O},>99 \%$ Technical Grade, VWR Chemicals) and iso-propanol (C3H8O, 99.5\%, anhydrous, Sigma-Aldrich). The delamination medium was prepared by measuring the required amount of acetone ( $\mathrm{v} / \mathrm{v} \%)$, which was diluted by iso-propanol ( $\mathrm{v} / \mathrm{v} \%)$. A total volume of $200 \mathrm{~mL}$ of the delamination medium was used. The beakers were sealed with parafilm. The beakers were heated to the target temperature by using a magnetic hot plate stirrer (Magnetic Stirrer, IKA $®$ RT10, Germany), and the agitation speed was set at $600 \mathrm{rpm}$. Agitation was conducted to ensure that all the solids were suspended in the solvent. WPB samples of known weight were then added into the delamination medium once the target temperature had been reached. Since the boiling point of acetone is $56^{\circ} \mathrm{C}$, the temperature range used for the experiments was set to $30-50^{\circ} \mathrm{C}$.

The experiments shown in Table I were performed for Samples A and B. Experiments A1, A6 and A11 were conducted to ascertain whether isopropanol can delaminate WPBs on its own and whether a higher temperature is required for delamination. Experiments A2-A5, A7-A10 and
A12-A15 were carried out to ascertain the effect of temperature and the acetone concentration $(\mathrm{v} / \mathrm{v} \%)$, whereas experiments A16-A20 were performed to study the effect of residence time on the delamination of WPBs.

After performing the delamination experiments for Samples A and B, the experiments shown in Table I for the Sample C were performed to investigate and verify the optimized parameter levels.

After each experimental run time, the separated aluminum and plastic layers were carefully collected from the delamination medium. The collected aluminum layers were dried overnight at room temperature and then weighed. The aluminum recovery was then calculated based on the wt.\% of the aluminum layer in each sample of WPBs varying between $10 \mathrm{wt} . \%$ and $15 \mathrm{wt} . \%$. The wt.\% of aluminum layer in Sample A was 15 wt.\%, in Sample B was 10 wt.\% and in Sample C was 13 wt.\%. The aluminum layers were then analyzed using a scanning electron microscope (SEM, Tescan Mira3 GM, Czech Republic) coupled with energydispersive spectroscopy (EDS, UltraDry $50 \mathrm{~mm}^{2}$ EDS detector and NSS software, Thermo Scientific, USA) wherever needed.

\section{RESULTS AND DISCUSSION}

\section{Characterization of WPB Samples}

The characterization of WPBs was performed using chemical analysis by employing inductively coupled plasma-optical emission spectroscopy (ICP-

Table I. Experiments and their parameter levels utilized in this study for WPB Sample A, B and C

\begin{tabular}{|c|c|c|c|c|}
\hline Experiment codes* & Temperature $\left({ }^{\circ} \mathbf{C}\right)$ & Time (min) & Acetone (v/v\%) & Iso-propanol (v/v\%) \\
\hline A1, B1 & 30 & 120 & 0 & 100 \\
\hline $\mathrm{A} 2, \mathrm{~B} 2$ & 30 & 120 & 25 & 75 \\
\hline A3, B3 & 30 & 120 & 50 & 50 \\
\hline $\mathrm{A} 4, \mathrm{~B} 4$ & 30 & 120 & 75 & 25 \\
\hline $\mathrm{A} 5, \mathrm{~B} 5$ & 30 & 120 & 100 & 0 \\
\hline $\mathrm{A} 6, \mathrm{~B} 6, \mathrm{C} 1$ & 40 & 120 & 0 & 100 \\
\hline $\mathrm{A} 7, \mathrm{~B} 7, \mathrm{C} 2$ & 40 & 120 & 25 & 75 \\
\hline $\mathrm{A} 8, \mathrm{~B} 8, \mathrm{C} 3$ & 40 & 120 & 50 & 50 \\
\hline $\mathrm{A} 9, \mathrm{~B} 9, \mathrm{C} 4$ & 40 & 120 & 75 & 25 \\
\hline $\mathrm{A} 10, \mathrm{~B} 10, \mathrm{C} 5$ & 40 & 120 & 100 & 0 \\
\hline $\mathrm{A} 11, \mathrm{~B} 11$ & 50 & 120 & 0 & 100 \\
\hline $\mathrm{A} 12, \mathrm{~B} 12$ & 50 & 120 & 25 & 75 \\
\hline $\mathrm{A} 13, \mathrm{~B} 13$ & 50 & 120 & 50 & 50 \\
\hline $\mathrm{A} 14, \mathrm{~B} 14$ & 50 & 120 & 75 & 25 \\
\hline A15, B15 & 50 & 120 & 100 & 0 \\
\hline $\mathrm{A} 16, \mathrm{~B} 16$ & 40 & 30 & 50 & 50 \\
\hline A17, B17 & 40 & 60 & 50 & 50 \\
\hline A18, B18 & 40 & 90 & 50 & 50 \\
\hline A19, B19 & 40 & 120 & 50 & 50 \\
\hline $\mathrm{A} 20, \mathrm{~B} 20$ & 40 & 120 & 50 & 50 \\
\hline
\end{tabular}

*Experiment codes for Sample B are B1-B20 and for Sample C are C1-C5. 
OES, PerkinElmer Optima 7100 DV, USA). The purity of the aluminum in samples A and B was $>$ 99.4\%. The results indicated that the aluminum layer constitutes $15 \mathrm{wt} \%$ and $10 \mathrm{wt} . \%$ of the total weight of Samples A and B, respectively. However, for Sample C, the aluminum layer constitutes 13 wt.\% of the total weight of the WPBs. The investigated WPB Samples A and B were characterized by FTIR (Fig. 3). The prominent distinctive bands found in the spectra were at a wavelength of $2906 \mathrm{~cm}^{-1}$, which represents $\mathrm{C}-\mathrm{H}$ stretching of the $\mathrm{CH}-\mathrm{Cl}$ group, and at $1425 \mathrm{~cm}^{-1}$ because of $\mathrm{CH}_{2}$ deformation; at $1234 \mathrm{~cm}^{-1}$, which demonstrates C-H deformation corresponding to a $\mathrm{CH}-\mathrm{Cl}$ group; at 960 $\mathrm{cm}^{-1}$, which displays the rocking of the $\mathrm{CH}_{2}$ group; and at $605 \mathrm{~cm}^{-1}$ because of $\mathrm{CH}-\mathrm{Cl}$ stretching. ${ }^{22-24}$ The FTIR analysis for the material used for the adhesion of aluminum and plastic layers closely matched the spectrum of poly-acrylic cyanoacrylate, as reported by Han et al. ${ }^{25}$ Hence, it can be concluded that the material used for the adhesion of aluminum and plastic layers was a poly-acrylic based glue.
Furthermore, the SEM micrographs showed the cross sections of Samples A and B in Fig. $4 a$ and b respectively. It was observed that in both samples there were two layers of plastics present in the forming film and a metallic layer of aluminum as the lidding material. As also shown in Fig. 4, the overall thickness of WPBs ranged from 280-365 $\mu \mathrm{m}$ and comprised two plastic layers (D1 and D2) in the range of 260-330 $\mu \mathrm{m}$ and an aluminum layer (D3) in the range of 20-35 $\mu \mathrm{m}$. Therefore, based on the FTIR results, the chemical composition of the forming films of blister packaging indicates the presence of PVC and PVDC. Additionally, according to further analysis of the FTIR results (Fig. 3) performed on Sample A (Plastic Layer 1) and Sample A (Plastic Layer 2), the best fitted results were with polyvinyl chloride/polyvinyl acetate copolymer with $10 \%$ polyvinyl acetate and polyvinylidene chloride/polymethyl methacrylate copolymer with $10 \%$ of polymethyl methacrylate, respectively. However, for Sample B (Plastic Layer 1) and Sample B (Plastic Layer 1), the best fitted results were with polyvinyl chloride/polyvinyl
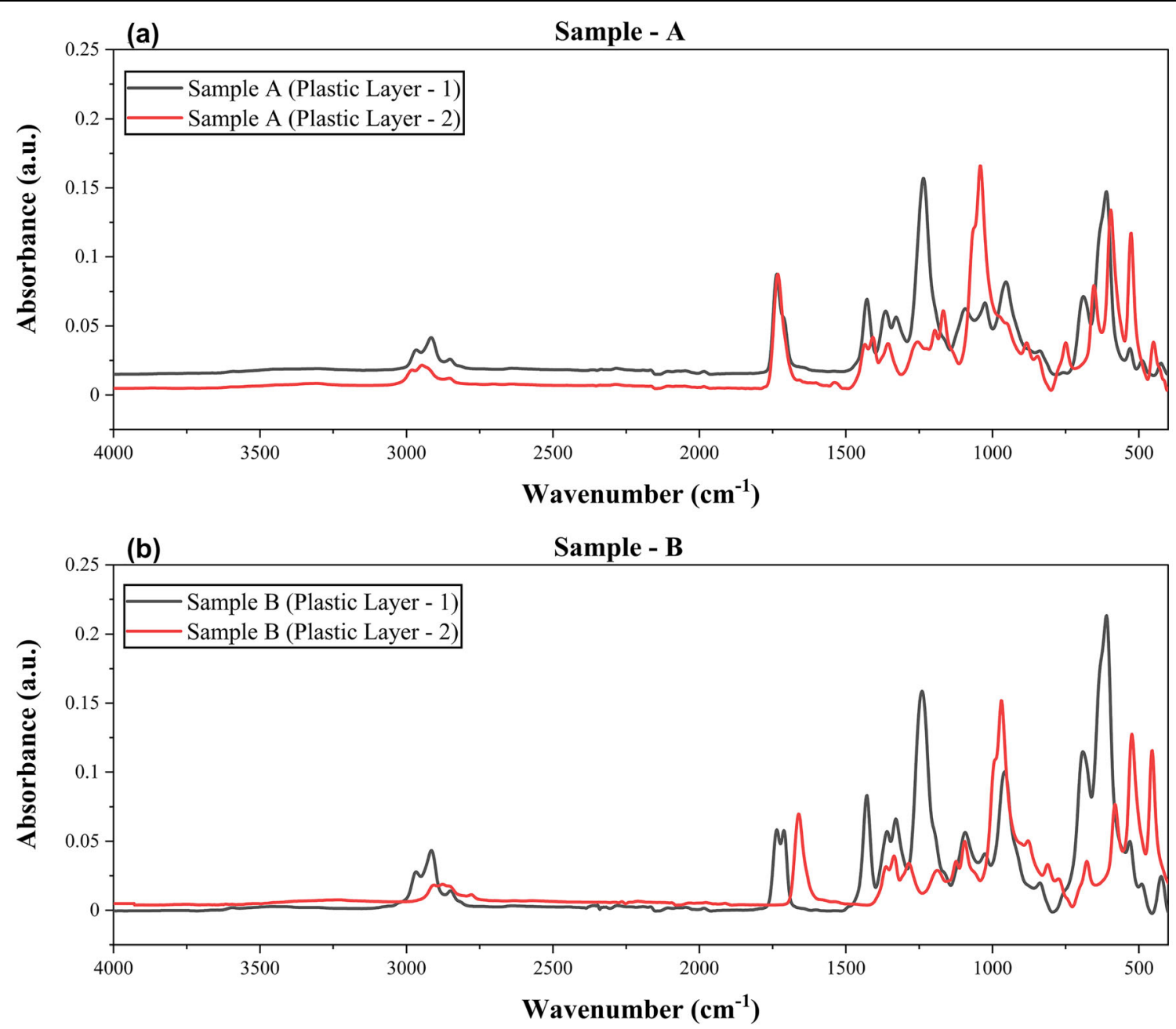

Fig. 3. FTIR spectra for (a) sample A and (b) sample B. 
(a)

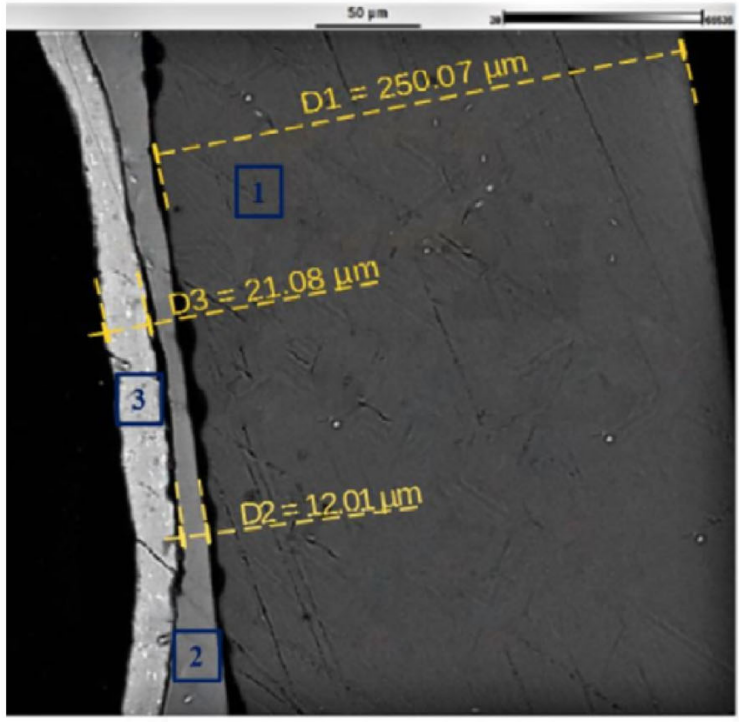

Sample A (b)

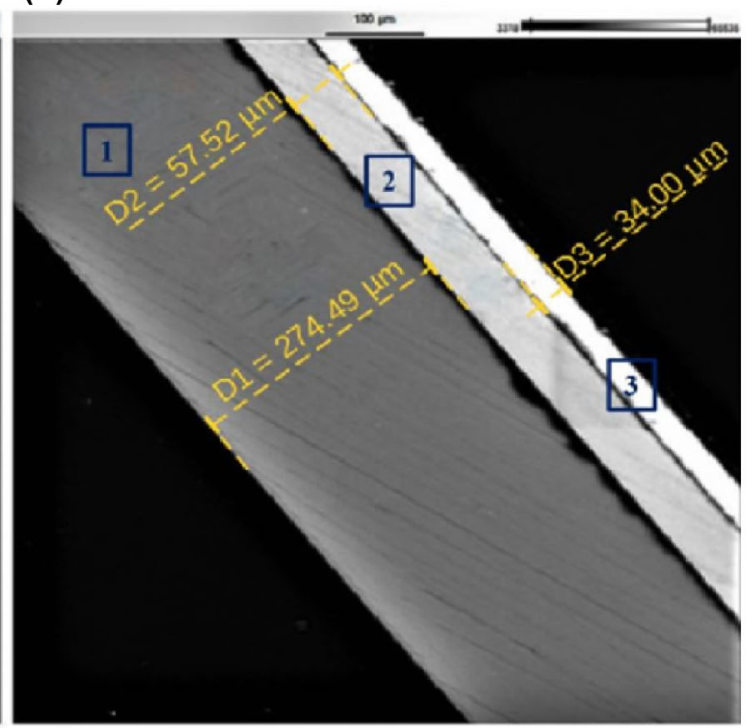

Sample B

Fig. 4. SEM micrographs of cross-sectional measurement and EDS analysis of plastic layers and metallic layer for (a) Sample A and (b) Sample B.

Table II. Elemental composition (wt.\%) for both Sample A and B (average composition)

\begin{tabular}{|c|c|c|c|c|c|c|c|c|c|c|c|c|c|c|}
\hline Element & $\mathbf{O}$ & $\mathbf{N a}$ & Al & $\mathbf{S i}$ & $\mathbf{P}$ & $\mathbf{S}$ & Cl & Zn & $\mathbf{C a}$ & $\mathbf{T i}$ & Pd & Sn & $\mathbf{F e}$ & Total \\
\hline \multicolumn{15}{|l|}{ Sample A } \\
\hline Spot 1 & 11.6 & 0.1 & 0.3 & 0.2 & - & 0.4 & 81.5 & - & 0.03 & - & 4.6 & 1.4 & - & 100 \\
\hline Spot 2 & 16.1 & 1.3 & 0.3 & 0.1 & 0.4 & 0.8 & 76.6 & 0.2 & - & - & 4.3 & - & - & 100 \\
\hline Spot 3 & 1.6 & - & 95.8 & 1.1 & - & 0.04 & 1.1 & - & 0.02 & - & - & - & 0.4 & 100 \\
\hline \multicolumn{15}{|l|}{ Sample B } \\
\hline Spot 1 & 15.8 & - & 0.5 & 0.2 & - & 0.4 & 73.0 & - & 0.1 & 4.6 & 4.6 & 0.9 & - & 100 \\
\hline Spot 2 & 12.5 & 1.6 & 0.3 & 0.1 & 0.4 & 0.8 & 80.1 & - & - & - & 4.3 & - & - & 100 \\
\hline Spot 3 & 1.8 & - & 97.3 & - & 0.1 & - & 0.1 & - & - & - & - & - & 0.8 & 100 \\
\hline
\end{tabular}

acetate copolymer with $2 \%$ polyvinyl acetate and polyvinyl chloride, respectively. The FTIR spectra before and after the delamination of Samples A, B and C closely matched the PVC and PVDC spectra (see supplementary Figs. S1, S2 and S3).

The elemental composition of the plastic and metallic layers as shown in Table II is the average composition of three points for each layer, i.e., Spot 1 for Sample A is an average of EDS performed for three different points on Plastic Layer 1 of Sample A. The elemental composition (wt.\%) also confirms that after delamination the metallic layer of all the samples mainly comprises aluminum (see supplementary Tables S1, S2 and S3). Moreover, the presence of a significant amount of chlorine in the plastic layers clearly suggests that the plastic layers are composed of PVC and PVDC.

\section{Effect of Acetone Concentration, Temperature and Time on Delamination}

Experiments $\mathrm{A} 1-\mathrm{A} 5$ at $30^{\circ} \mathrm{C}, \mathrm{A} 6-\mathrm{A} 10$ at $40^{\circ} \mathrm{C}$ and $\mathrm{A} 11-\mathrm{A} 15$ at $50^{\circ} \mathrm{C}$ were performed on Sample A. The run time for all the experiments was $120 \mathrm{~min}$. No delamination was observed for acetone concentrations $<50 \mathrm{v} / \mathrm{v} \%$, i.e., A1, A2, A6, A7, A11 and A12. Also, no delamination was observed at $50 \mathrm{v} / \mathrm{v} \%$ acetone concentration, i.e., A3. However, for all the other experimental operating conditions, i.e., A4, A5, A8-A10 and A13-A15, total delamination was achieved, as shown in Fig. 5a.

The experiments on Sample B were performed in similar operating conditions, i.e., B1-B5, B6-B10 and B11-B15. For Sample B, delamination was observed at acetone concentrations of $50 \mathrm{v} / \mathrm{v} \%$ and higher, i.e., B3-B5, B8-B10 and B13-B15. However, for Sample B, delamination was also observed at an acetone concentration of $25 \mathrm{v} / \mathrm{v} \%$ at $50^{\circ} \mathrm{C}$, i.e., 

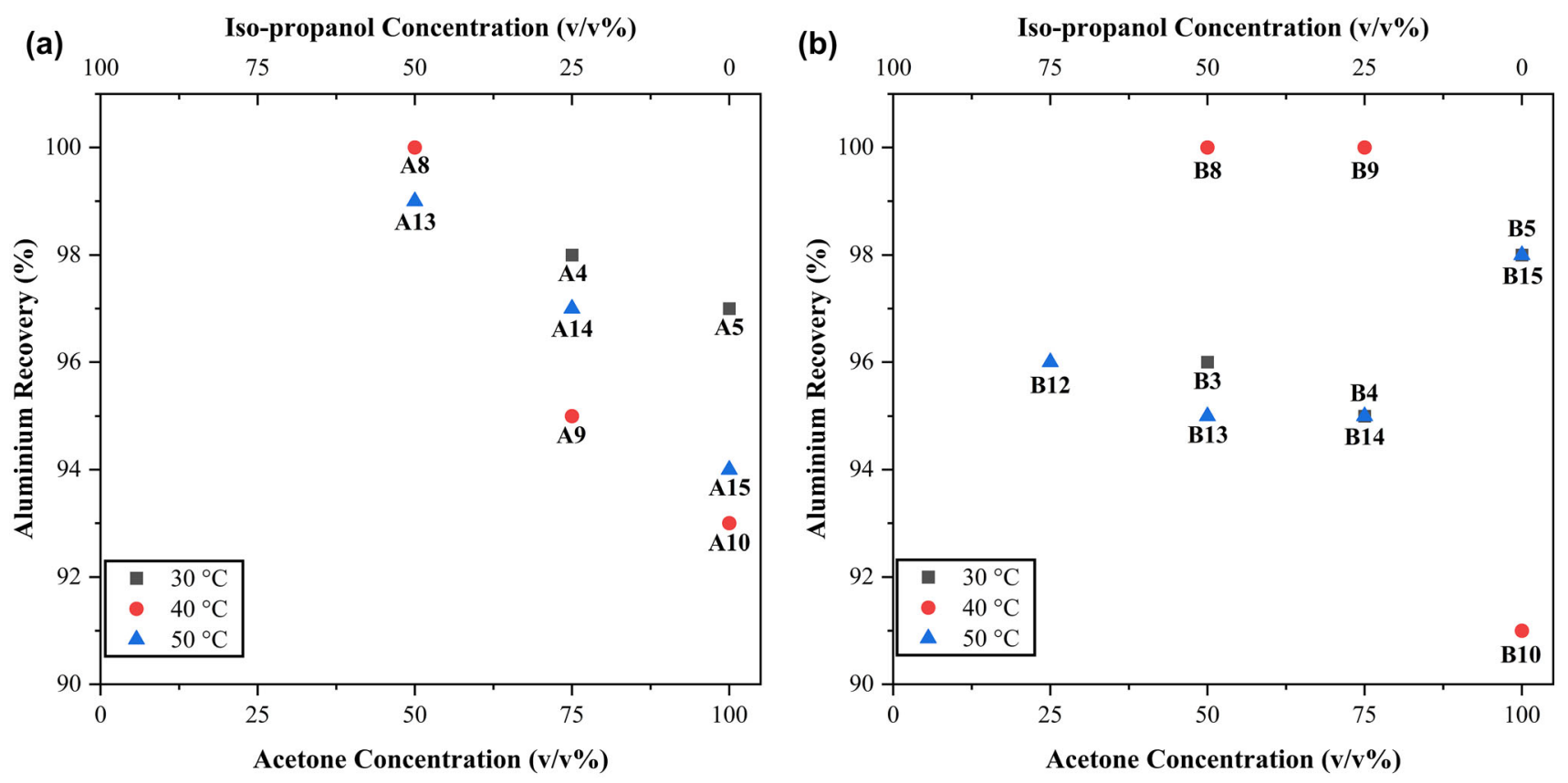

Fig. 5. Aluminum recovery at different acetone concentrations for (a) Sample A and (b) Sample B.

B12, as shown in Fig. 5b. The total delamination of WPBs with $100 \%$ aluminum recovery was attained at the acetone concentration of $50 \mathrm{v} / \mathrm{v} \%$ at $40^{\circ} \mathrm{C}$ for both Samples A and B.

The investigated process temperatures were 30 , 40 and $50^{\circ} \mathrm{C}$ for both Samples A and B, as shown in Fig. 5. The operating temperature had a significant effect on both the delamination process as well as on the recovery of aluminum from the delaminated samples. A $100 \%$ recovery of aluminum was achieved for an acetone concentration of $50 \mathrm{v} / \mathrm{v} \%$ and at a temperature of $40^{\circ} \mathrm{C}$ for both Samples A and B, i.e., A8 and B8. However, with an increase in acetone concentration at $40^{\circ} \mathrm{C}$, the aluminum recovery decreased. The higher temperature, i.e., $>40^{\circ} \mathrm{C}$, facilitates the decomposition of plastic layers and adhesive. Moreover, when higher concentration of acetone, i.e., $>50 \mathrm{v} / \mathrm{v} \%$, is used, the plastic layers and adhesive start to decompose and remain as residual within the solvent, whilst during the overnight drying of samples in atmospheric conditions, the residuals from the decomposed plastic and adhesive tend to reattach at aluminum surface resulting the decrease in aluminum recovery.

The effect of residence time, i.e., $30 \mathrm{~min}, 60 \mathrm{~min}$, $90 \mathrm{~min}$ and $120 \mathrm{~min}$, on the delamination of WPBs and aluminum recovery was studied at $40^{\circ} \mathrm{C}$ and with an acetone concentration of $50 \mathrm{v} / \mathrm{v} \%$. The experiment codes for Sample A were A16-A19 and for Sample B were B16-B19 (Table I). No delamination observed at $30 \mathrm{~min}$ for Sample A. However, for Sample B, the first plastic layer was completely delaminated within $30 \mathrm{~min}$, whereas for Sample A, the first plastic layer was delaminated after $60 \mathrm{~min}$. It was also observed that the second plastic layer was partially delaminated for both Samples A and B if the residence time was $<120 \mathrm{~min}$. After $120 \mathrm{~min}$, the total delamination of both plastic layers along with aluminum was achieved. The results from the residence time experiments suggest that the time required to penetrate through different plastic layers varies because of the plastic layer structure, chemical composition and thickness of plastic layers. Therefore, delamination behavior is different for Samples A and B. However, the time required for total delamination for both Sample A and B was 120 min.

Experiments A20 and B20 (Table I) were performed to ascertain whether these investigated experimental conditions were the optimal operating parameters for the delamination of Samples A and B. Based on the results, it is suggested that the mechanism for the delamination process involves the depolymerization of the acrylic adhesive present between the aluminum and plastic layers of WPBs in the presence of a delaminating agent and that the depolymerized monomer remains in the medium. The acetone swells the plastics layers but reaches a limit because of the crosslinked structure of the plastic layers.

According to the studies reported by Summers and Rabinovitch ${ }^{19}$ and Perry et al., ${ }^{26}$ this is due to the diffusion of acetone through the amorphous plastic but not the crystallites/crystalline PVC, thereby constructing porous channels for the acetone to diffuse toward the interface between the plastic layers and aluminum glued together with acrylic adhesive. As the acetone is diffused toward the interface, it allows the depolymerization of the acrylic adhesive, leading to delamination. ${ }^{19,26}$ Han et al. $^{25}$ mentioned a similar phenomenon and reported that various poly-alkyl-cyanoacrylate 
(PACA) polymers used as adhesives in the pharmaceutical industry undergo rapid depolymerization in the presence of acetone. Bruze et al. ${ }^{27}$ also reported similar findings while performing patch testing for occupational allergic contact dermatitis for ethylcyanoacrylate, which is used as an adhesive. They reported that results were false-negative for a test when a different acetone concentration was added into the chambers for the Finn Chambers ${ }^{\circledR}$ patch test technique since, in Finn Chambers ${ }^{\circledR}$, the chambers are constructed from aluminum and filled with the allergen, i.e., ethyl-cyanoacrylate adhesive dissolved in acetone, for testing the allergic reaction. After 2 days, the results for allergic reactions were false negative because of the denaturing of the ethyl-cyanoacrylate. Hence, when comparing the study from Bruze et al., ${ }^{27}$ it is suggested that a similar mechanism occurs during the delamination of WPBs. Therefore, the results elucidate the reason for required retention time, temperature and acetone concentration for the total delamination of different WPBs. Moreover, the results also show that the structure and chemical composition (amount of amorphous and crystalline PVC) of plastic layers are different in Sample A and Sample $\mathrm{B}$, which is similar to the claims made by Summers and Rabinovitch ${ }^{19}$ that acetone swells the PVC because of the penetration of acetone into the amorphous PVC without altering the crystalline PVC.

\section{Verification of Parameter Optimization, Reuse of Solvent and Laboratory Scale-Up}

To ascertain that the parameters were optimized for any type of WPBs, delamination was performed for sample $\mathrm{C}$ at $40^{\circ} \mathrm{C}$ for $120 \mathrm{~min}$ with $600 \mathrm{rpm}$ agitation. No delamination for sample $\mathrm{C}$ was

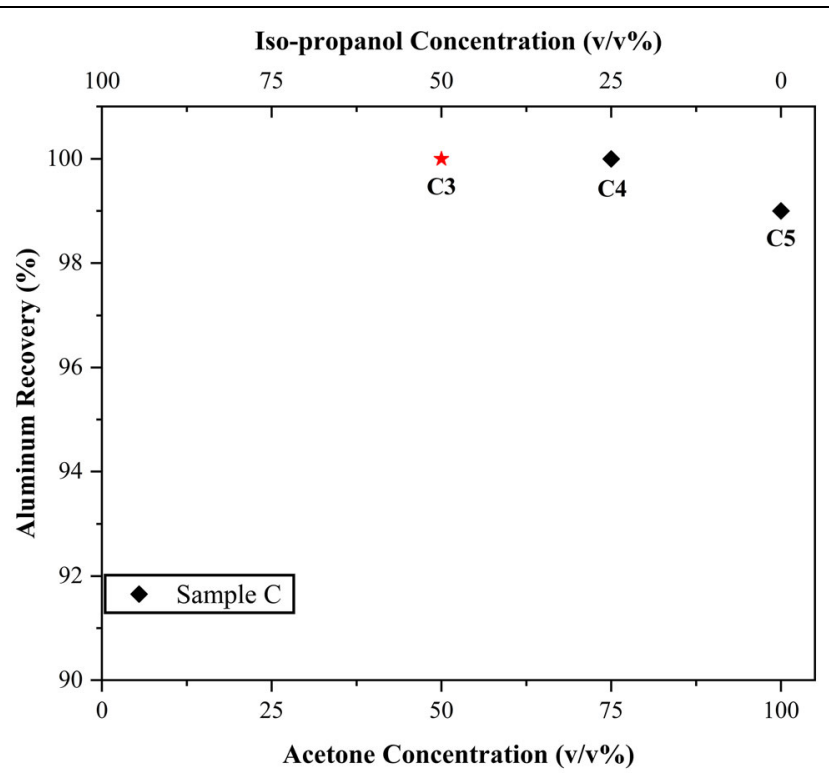

Fig. 6. Parameter optimization experiments and aluminum recovery for Sample C. observed for experiments with an acetone concentration $<50 \mathrm{v} / \mathrm{v} \%$, i.e., C1 and C2. However, for an acetone concentration $\geq 50 \mathrm{v} / \mathrm{v} \%$, total delamination of sample C was achieved, i.e., C3-C5, with 100\% aluminum recovery at $\mathrm{C} 3$ and $\mathrm{C} 4$, as shown in Fig. 6. Hence, the parameters for experiment C3 were suggested as the optimized parameters because of the lower acetone concentration compared to $\mathrm{C} 4$ and $\mathrm{C} 5$, allowing better recovery of aluminum from all of sample $\mathrm{C}$.

The recovery and reuse of delaminating agent were carried out to establish and reduce the amount of solvent waste generated. Additionally, to support the principles of circular economy and green chemistry along with the potential commercial application, the reuse of the delaminating agent was studied by reusing it six times. The reuse of the delaminating agent was carried out in optimized conditions for sample $\mathrm{C}$, i.e., at $40^{\circ} \mathrm{C}$, for $120 \mathrm{~min}$ at $600 \mathrm{rpm}$ agitation. The initial volume of delaminating agent was $200 \mathrm{~mL}$ with an acetone concentration of $50 \mathrm{v} / \mathrm{v} \%$. After each cycle, the remaining volume of the delaminating agent was measured and visually inspected. The loss in volume after each cycle was $7 \pm 2 \mathrm{~mL}$. The loss in volume after each cycle was observed because of the volatile nature of the delaminating agent and because the experimental setup was not hermetically sealed. Although the time required for total delamination remained unchanged, the visual inspection performed after each cycle revealed an accumulation of residuals.

Furthermore, to ascertain the laboratory scale up of the delamination process, four pieceseach from Sample A, B and C were used for the experiments under optimized parameters, i.e., at $40^{\circ} \mathrm{C}$ for 120 $\mathrm{min}$, at $600 \mathrm{rpm}$ agitation and an acetone concentration of $50 \mathrm{v} / \mathrm{v} \%$. However, the total volume of the delaminating agent used was $800 \mathrm{~mL}$ with overhead stirring to ensure that the WPBs were well suspended in the delaminating agent. The laboratory scale-up experiment resulted in total delamination of all 12 WPBs (four pieces each from Sample A, B and $\mathrm{C}$ ), with $100 \%$ aluminum recovery at the end of laboratory scale-up experiment.

\section{Discussion}

The delamination experiments showed that aluminum separation from the plastic fraction was achieved at optimized operating parameters for different types of WPBs. However, for feasibility of the process, effective solid liquid separation of aluminum and plastics (solid) from the delaminating agent (liquid) is needed, e.g., by using a horizontal belt vacuum filtration unit. The separated delaminating agent can be reused, while the aluminum and plastics can be fed for drying (optional) prior to further processing. The segregation of aluminum and plastic can be achieved through an eddy current separator. In the current study, the 


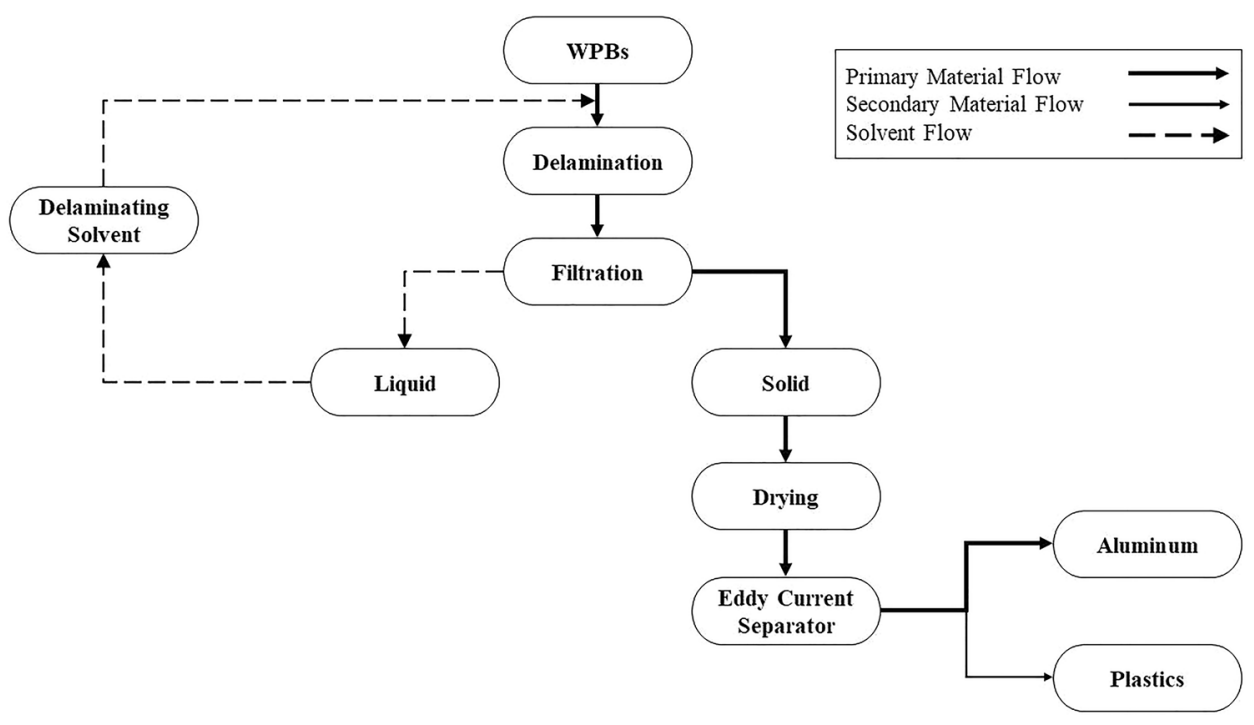

Fig. 7. Overall indicative flowsheet for delamination of WPBs for recycling.

application of eddy current separation was ensured by using a laboratory-scale eddy current separator for the segregation of aluminum and plastics from delaminated WPBs. The eddy current separator provided complete segregation of aluminum and plastics. The separated aluminum can be recycled via a secondary aluminum smelter, whereas plastics could potentially be subjected to further processing. An overall indicative flowsheet for the entire process is shown in Fig. 7.

The advantages of the proposed process are high aluminum separation $(\sim 100 \%)$ using green solvent, at low temperature and with heterogenous feed material, i.e., different WPBs. The process requires no pre-processing of the raw material. However, acetone and iso-propanol are highly volatile and flammable and can cause mild irritation if exposed to them for a longer period. Once these issues are resolved, the then proposed methodology may provide increased efficiency for aluminum recycling from WPBs and can be both environmentally and economically attractive in an industrially scaled-up operation.

\section{CONCLUSION}

The current study presents, for the first time to our knowledge, a novel application of the delamination technique for separating the aluminum layer from the plastic layer in WPBs. This allows recycling of aluminum and potential plastics obtained from the delamination of WPBs. The delamination process for WPBs was successfully conducted by using acetone and iso-propanol $(50 \mathrm{v} / \mathrm{v} \%)$ as the delaminating agent. The operating parameters were optimized for a heterogeneous feed of WPBs. The delamination process occurred primarily in two stages, i.e., diffusion of delaminating agent through the plastics to the interface and then depolymerization of the adhesive. Total delamination was obtained for all the investigated samples, A, B and $\mathrm{C}$, in the optimized parameters, i.e., at $40^{\circ} \mathrm{C}$ with an acetone concentration of $50 \mathrm{v} / \mathrm{v} \%(200 \mathrm{~mL})$ and retention time of $120 \mathrm{~min}$.

\section{ACKNOWLEDGEMENTS}

The authors acknowledge the SUDDEN Project (Decision No. 320219) funded by the Strategic Research Council at the Academy of Finland and Pharmac Finland Oy is gratefully acknowledged for providing the support and WPBs investigated in this study. This work also made use of RawMatTERS Finland Infrastructure (RAMI) funded by the Academy of Finland.

\section{FUNDING}

Open Access funding provided by Aalto University.

\section{CONFLICT OF INTEREST}

On behalf of all authors, the corresponding author states that there is no conflict of interest.

\section{OPEN ACCESS}

This article is licensed under a Creative Commons Attribution 4.0 International License, which permits use, sharing, adaptation, distribution and reproduction in any medium or format, as long as you give appropriate credit to the original author(s) and the source, provide a link to the Creative Commons licence, and indicate if changes were made. The images or other third party material in this article are included in the article's Creative Commons licence, unless indicated otherwise in a credit line to the material. If material is not included in the article's Creative Commons licence and your intended use is not permitted by statutory regulation or exceeds the permitted use, you will 
need to obtain permission directly from the copyright holder. To view a copy of this licence, visit $h$ ttp://creativecommons.org/licenses/by/4.0/.

\section{SUPPLEMENTARY INFORMATION}

The online version contains supplementary material available at https://doi.org/10.1007/s11837021-05038-6.

\section{REFERENCES}

1. R. Pilchik, Pharm. Technol. 24(11), 68 (2000).

2. J. Nieminen, I. Anugwom, M. Kallioinen, and M. Mänttäri, Waste Manag. 107, 20. https://doi.org/10.1016/j.wasman.20 20.03.014 (2020).

3. K. Hänsch and K. Kinkel, J. Environ. Law 7(2), 323. http s://doi.org/10.1093/jel/7.2.323 (1995).

4. G. Kale, T. Kijchavengkul, R. Auras, M. Rubino, S.E. Selke, and S.P. Singh, Macromol. Biosci. 7(3), 255. https://doi.org/ 10.1002/mabi.200600168 (2007).

5. V. Agarwal, P. Halli, S. Helin, F. Tesfaye, and M. Lundström, A.C.S. Sustain. Chem. Eng. 8(10), 4137. https://doi. org/10.1021/acssuschemeng.9b06810 (2020).

6. B.K. Lee, M.J. Ellenbecker, and R. Moure-Eraso, Waste Manag. 22(5), 461. https://doi.org/10.1016/S0956-053X(02)0 0006-5 (2002)

7. C. Wang, H. Wang, and Y. Liu, J. Clean. Prod. 102, 378. h ttps://doi.org/10.1016/j.jclepro.2015.04.067 (2015).

8. M.F. Ali and M.N. Siddiqui, J. Anal. Appl. Pyrolysis 74(1-2), 282. https://doi.org/10.1016/j.jaap.2004.12.010 (2005).

9. C.H. Park, H.S. Jeon, and J.K. Park, J. Hazard. Mater. 144(1-2), 470. https://doi.org/10.1016/j.jhazmat.2006.10.060 (2007).

10. G. Montaudo and C. Puglisi, Polym. Degrad. Stab. 33(2), 229. https://doi.org/10.1016/0141-3910(91)90019-N (1991).

11. M.Y. Wey, L.J. Yu, and S.I. Jou, J. Hazard. Mater. 60(3), 259. https://doi.org/10.1016/S0304-3894(98)00100-9 (1998).

12. A. Kitazawa, T. Amagai, and T. Ohura, Environ. Sci. Technol. 40(15), 4592. https://doi.org/10.1021/es0602703 (2006).

13. M. Sadat-Shojai and G.R. Bakhshandeh, Polym. Degrad. Stab. 96(4), 404. https://doi.org/10.1016/j.polymdegradstab. 2010.12.001 (2011)
14. F.P. La Mantia, Recycling of PVC and Mixed Plastic Waste (ChemTec Publishing, Toronto, 1996), pp 63-76.

15. V. Gente, F. La Marca, F. Lucci, and P. Massacci, Waste Manag. 23(10), 951. https://doi.org/10.1016/S0956-053X(03) 00088-6 (2003).

16. V. Gente, F. La Marca, F. Lucci, P. Massacci, and E. Pani, Waste Manag. 24(7), 663. https://doi.org/10.1016/j.wasman. 2004.03.005 (2004).

17. C.-Q. Wang, H. Wang, G.-H. Gu, J.-G. Fu, Y.-N. Liu, and J. Cent, South Univ. 22(12), 4545. https://doi.org/10.1007/s11 771-015-3004-x (2015).

18. S. Yousef, T. Mumladze, M. Tatariants, R. Kriūkienè, V. Makarevicius, R. Bendikiene, and G. Denafas, J. Clean. Prod. 197(1), 379. https://doi.org/10.1016/j.jclepro.2018.06.1 54 (2018).

19. J.W. Summers and E.B. Rabinovitch, J. Macromol. Sci. Part B 20(2), 219. https://doi.org/10.1080/00222348108219439 (1981).

20. C.D. Papaspyrides and S.G. Tingas, Food Addit. Contam. 15(6), 681. https://doi.org/10.1080/02652039809374698 (1998).

21. O. Helmboldt, L.K. Hudson, C. Misra, K. Wefers, W. Heck, H. Stark, M. Danner, and N. Rösch, Ullmann's Encycl. Ind. Chem. 2, 569. https://doi.org/10.1002/14356007.a01_527.pub 2 (2007).

22. M. Beltrán and A. Marcilla, Eur. Polym. J. 33(7), 1135. h ttps://doi.org/10.1016/S0014-3057(97)00001-3 (1997).

23. A.H. Kuptsov and G.N. Zhizhin, Handbook of Fourier Transform Raman and Infrared Spectra of Polymers (Elsevier, Amsterdam, 1998), pp 48-50.

24. T.M.C. Pereira, J.A.Q. Júnior, R.S. Ortiz, W.F.C. Rocha, D.C. Endringer, P.R. Filgueiras, R.J. Poppi, and W. Romão, Anal. Methods 6(8), 2722. https://doi.org/10.1039/C3A Y41457D (2014).

25. M.G. Han, S. Kim, and S.X. Liu, Polym. Degrad. Stab. 93(7), 1243. https://doi.org/10.1016/j.polymdegradstab.2008.04.012 (2008).

26. K.L. Perry, P.J. McDonald, and A.S. Clough, Magn. Reson. Imaging 12(2), 217. https://doi.org/10.1016/0730-725X(94)9 1521-0 (1994).

27. M. Bruze, B. Björkner, and J.P. Lepoittevin, Occupational allergic contact dermatitis from ethyl cyanoacrylate. Contact Dermat. 32(3), 156. https://doi.org/10.1111/j.1600-0536. 1995.tb00806.x (1995).

Publisher's Note Springer Nature remains neutral with regard to jurisdictional claims in published maps and institutional affiliations. 